\title{
ENDOGENOUS SYNTHESIS OF PREBIOTIC ORGANIC
} MOLECULES

\author{
Stanley L. Miller \\ Department of Chemistry and Binchemistry \\ University of Calitomia, San Diego \\ La Jolla, Callifornia 92(193-(1506, USA
}

The necessary condition for the synthesis of organic compounds on the primitive earth is the presence of reducing conditions. This means an atmosphere of $\mathrm{CH}_{4}, \mathrm{CO}$, or $\mathrm{CO}_{2}+\mathrm{H}_{2}$. The atmospheric nitrogen can be $\mathrm{N}_{2}$ with a trace of $\mathrm{NH}_{3}$, but $\mathrm{NH}_{4}^{+}$is needed in the ocean at least for amino acid synthesis. Many attempts have heen made to use $\mathrm{CO}_{2}+\mathrm{H}_{2} \mathrm{O}$ atmospheres for prebiotic synthesis, but these give at best extremely low yields of organic compounds, except in the piesence of $\mathrm{H}_{2}$. Even strong reducing agents such as $\mathrm{FeS}+\mathrm{H}_{2} \mathrm{~S}$ or the mineral assemblages of the submarine vents fuil to give significant yields of organic compounds with $\mathrm{CO}_{2}$. There appears to be a high kinetic banier to the non-biological reduction of $\mathrm{CO}_{2}$ at low temperatures using geological reducing agents.

The most abundant source of energy for prebiotic synthesis is ultraviolet light followed by electric discharges, with electric discharges being more efficient, although it is not clear which was the important energy source. A reasonable rate of symthesis of amino acids firom these sources under relatively reducing conditions is about 100$)$ nmoles $\mathrm{cm}^{-2} \mathrm{yr}^{-1}$ of HCN, 10 nmoles $\mathrm{cm}^{-2} \mathrm{yr}^{-1}$ of amino acids or 0.10 moles $\mathrm{cm}^{-2}$ of amino acids in $10^{7} \mathrm{yrs}$. This would give a concentration of $3 \times 10^{-4} \mathrm{M}$ in an ocean of the present size (3)( ) liters $\mathrm{cm}^{-2}$ ). The amino acids cannot accumulate over a longer period becuuse the entire ocean passes through the $350^{\circ} \mathrm{C}$ submaline vents in $10^{7} \mathrm{yrs}$, which decomposes all the organic compounds. Less reducing conditions such as $\mathrm{CO} \mathrm{rCO}_{2}$ atmospheres with only small amounts of $\mathrm{H}_{2}$ give considerably less $\mathrm{HCN}$ and $\mathrm{H}_{2} \mathrm{CO}$ with electric discharges. These compounds are central to prebiotic syntheses. Photochemical process would also make signiticant contributions. In an atmosphere of $\mathrm{CO}_{2}, \mathrm{~N}_{2}$, and $\mathrm{H}_{2} \mathrm{O}$ with no $\mathrm{H}_{2}$, the production rates of $\mathrm{HCN}$ and $\mathrm{H}_{2} \mathrm{CO}$ would be very low, 0.001 or less than that of a relatively reducing atmosphere. The concentration of organic compounds under these non-reducing conditions would be so low that there is doubt whether the concentration mechanism would be adequate for further steps toward the origin of life.

A number of workers have calculated the intlux of comets and meteorites on the primitive earth as a source of organic compounds. Some of the amino acids from the meteorite proposed to have hit the earth 
$65 \times 10^{6}$ yrs ago have heen detected at the Crelaceous/Tertiary boundary sediments.

The problem with proposing a large scale input of organic compounds from meteorites and comets is that they must survive passage through the atmosphere and the impact. There are some processes that would allow survival such as showers of entimeter to meter sized meteorites and various derodymamic braking processes for larger objects. Even if all the input organic compounds survived, the amounts would be small relative to the earth hased syntheses, except for the unfavorable case of a nonreducing atmosphere.

The earth based syntheses are continuous processes since much of the carbon from decomposed organic compounds can be recycled. The organic input firom comets and metemites is equivalent to a one time syntheses, and the destructive processes in the hydrothermal vents would remove these compounds on the average in $10^{7}$ yrs or less. There are many other decomposition processes besides the vents for organic compounds. If it is assumed that the input rate was sufficient to overcome these destructive processes, then ton much carbon and water, especially from comets, would have heen added to the earth from comets and meteorites. We conclude that while some organic material was added to the earth fiom comets and meteorites the amount available from these sources at a given time was at hest only a few percent of that from earth based syntheses under reducing conditions.
EXTRATERRESTRIAL PREBIOTIC MOLECULE!

\author{
Five College Radio \\ Massachuse \\ (irvine
}

Organic material appears ts The interstellar medium. spectroscopic signature 0 emission from warm dust stars and planets form fror provides at minimum an planetary material; in fa thermodynamics of colla ionization which controls $c$ "stickiness" of accreting gr some of this interstellar $\mathrm{n}$. the primitive Earth.

The interstellar medium $i$ Galaxy. Here more than 16 have been securely identi highest radio frequencies. molecular weights up to al of emission features wil evidence for significantly aromatics, although preci. yet been obtained. The lou to very large isotopic frac ratio in a specific molecule can be 1000 to $10,000 \mathrm{t}$ isotopic signature appea carbonaceous chondritic $m$. Simple organics such as $n$ of the giant planets and of is generally thought to $b_{1}$ system atmosphereless bo the asteroid belt. Such a: carbonaceous chondrites, organic material. This inc of terrestrial kerogen, as acids, carboxylic acids, $s$ nitrogen heterocycles, alc and polar hydrocarbons, a 